\title{
Molecular Ordering at the Interface Between Liquid Water and Rutile $\mathrm{TiO}_{2}(110)$
}

\author{
By Giulia Serrano, ${ }^{\dagger}$ Beatrice Bonanni, Marco Di Giovannantonio, ${ }^{\ddagger}$ Tomasz Kosmala, \\ Michael Schmid, Ulrike Diebold, Aldo Di Carlo, Jun Cheng, Joost VandeVondele, \\ Klaus Wandelt, and Claudio Goletti*
}

Keywords: DFT-MD simulations, scanning probe techniques, solid-liquid interface, $\mathrm{TiO}_{2}$, water adsorption

\begin{abstract}
The pivotal importance of $\mathrm{TiO}_{2}$ as a technological material involves most applications in an aqueous environment, but the single-crystal $\mathrm{TiO}_{2} /$ bulk-water interfaces are almost completely unexplored, since up to date solid/liquid interfaces are more difficult to access than surfaces in ultrahigh vacuum (UHV). Only a few techniques (as scanning probe microscopy) offer the opportunity to explore these systems under realistic conditions. The rutile $\mathrm{TiO}_{2}(110)$ surface immersed in high-purity water is studied by in situ scanning tunneling microscopy. The large-scale surface morphology as obtained after preparation under UHV conditions remains unchanged upon prolonged exposure to bulk water. Moreover, in contrast to UHV, atomically resolved images show a twofold periodicity along the [001] direction, indicative of an ordered structure resulting from the hydration layer. This is consistent with density-functional theory based molecular dynamics simulations where neighboring interfacial molecules of the first water layer in contact with the bulk liquid form dimers. By contrast, this dimerization is not observed for a single adsorbed water monolayer, i.e., in the absence of bulk water.
\end{abstract}

Dr. G. Serrano, Dr. B. Bonanni, Dr. M. Di Giovannantonio, T. Kosmala, Prof. C. Goletti, Department of Physics and CNISM, Università degli Studi di Roma "Tor Vergata", Via della Ricerca Scientifica 1, I-00133 Rome, Italy

T. Kosmala, Prof. K. Wandelt, Institut für Physikalische und Theoretische Chemie, University of Bonn, Wegeler Str. 12 53115 Bonn, Germany

Dr. M. Schmid, Prof. U. Diebold, Institute of Applied Physics, Vienna University of Technology, Wiedner Hauptstrasse 8-10/134 A-1040 Vienna, Austria

Prof. A. Di Carlo, Department of Electronic Engineering and CHOSE, Università degli Studi di Roma "Tor Vergata", Via del Politecnico 1 I-00133 Rome, Italy

Dr. J. Cheng, Department of Chemistry, University of Aberdeen, Meston Building, Meston Walk Aberdeen AB24 3 UE, UK

Prof. J. VandeVondele, Department of Materials, ETH Zürich, Wolfgang-Pauli-Strasse 27, CH-8093 Zürich, Switzerland

[†] Present address: Institute of Semiconductor and Solid State Physics, Johannes Kepler University, Altenbergerstr. 69, 4040 Linz, Austria

[†] Present address: Istituto di Struttura della Materia (ISM), Consiglio Nazionale delle Ricerche (CNR), Via Fosso del Cavaliere 100, 00133 Rome, Italy

\section{Introduction}

The fundamental physical and chemical surface properties of titanium dioxide have been studied extensively in recent years. This high interest is stimulated by the important role of surfacerelated processes in a wide range of environmental and energy applications of $\mathrm{TiO}_{2}$, such as photoelectrolysis, photocatalysis, gas sensing, and photovoltaics. ${ }^{[1]}$ In many of these applications, $\mathrm{TiO}_{2}$ is immersed in water. Thus, a detailed understanding of the atomic scale mechanisms of processes occurring at the interface in water would represent a notable breakthrough in materials science. So far single crystal $\mathrm{TiO}_{2}$ surfaces have mostly been studied in ultrahigh vacuum (UHV), ${ }^{[2]}$ or under ambientpressure conditions, ${ }^{[3]}$ while in the literature only a very few studies exist about a $\mathrm{TiO}_{2}$ crystal surface immersed in a liquid phase. ${ }^{[4]}$ Particular attention has been paid to the rutile (110) surface in its most stable form, i.e., with a $(1 \times 1)$ termination, which is considered to be a prototypical model system in the surface science of metal oxides. The structure of this surface has been clarified in UHV, ${ }^{[2]}$ and the presence and the role of defects that are introduced by sputtering and annealing cycles have been discussed extensively. In addition, the adsorption of a large variety of molecules on this surface has been investigated, e.g., water, oxygen, carboxylic acids, alcohols, and other organic compounds. ${ }^{[\mathrm{b}, 5]}$ 


\section{ADVANCEP MateralaLs INTERFACES}

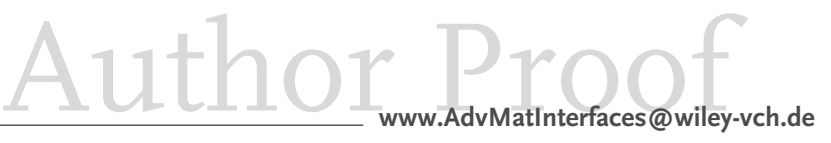

The interaction of this surface with water molecules under vacuum conditions appears to be a necessary first step to understand the surface chemistry in the liquid environment. However, surface processes in vacuum might be considerably different from those occurring at the solid/liquid interface. ${ }^{[6]}$ The scarcity of techniques that are capable of investigating solid surfaces immersed in a liquid represents a significant impairment in extending fundamental research to this more realistic environment.

The competitive molecular and dissociative adsorption of $\mathrm{H}_{2} \mathrm{O}$ molecules on a rutile (110) surface is a controversial issue. UHV studies of water adsorption have shown the role of defects: water dissociates at $\mathrm{O}$ vacancies and at step edges. ${ }^{[7-9]}$ Scanning tunneling microcopy (STM) in UHV showed that the first monolayer (ML) of water in direct contact with the surface adsorbs on undercoordinated $\mathrm{Ti}_{5 \mathrm{c}}$ atoms which form $\mathrm{1D}$ rows ${ }^{[10,11]}$ and provided evidence for the formation of isolated water dimers that diffuse faster than the monomers. ${ }^{[12]}$ Experiments and theoretical results are contradictory in whether or not the first layer molecules dissociate at the nondefective surface..$^{[9,10,12-20]}$

The situation is even more complicated when one considers a rutile $\mathrm{TiO}_{2}(110)$ surface immersed in liquid water. This system was modeled in multiple theoretical works. ${ }^{[17,20-25]}$ While all studies agree that the first layer of bulk water, in direct contact with the surface, is strongly bound, the degree of molecular dissociation is uncertain. From near-ambient X-ray photoelectron spectroscopy studies ${ }^{[18]}$ it was concluded that hydroxyl groups resulting from water dissociation at $O$ vacancies strongly affect adsorption within the first ML. Seminal X-ray diffraction studies ${ }^{[2,27]}$ of rutile (110) immersed in bulk water indicated a vertical ordering of the MLs as well as lateral ordering of the first hydrogen-bonded layer. Molecular dynamics (MD) simulations yielded conflicting results about the lateral arrangement of this layer. ${ }^{[20,28]}$ A UHV-STM study of $\mathrm{TiO}_{2}(110)$ before and after dipping the sample in water indicated that the surface might not be stable. ${ }^{[29]}$ This was also observed with atomic force microscope measurements in liquid. ${ }^{[30]}$

We report herein high-resolution STM images of the rutile $\mathrm{TiO}_{2}(110)$ surface in high-purity water. To our knowledge, this is the first STM investigation of this relevant surface in bulk water. We found that the observed surface structure with a distinct step-terrace morphology remains stable once immersed in liquid water. Interestingly, an ordered superstructure is also observed in high-resolution STM that indicates ordering of water molecules at the solid/liquid interface. Density functional theory-based molecular dynamics (DFT-MD) simulations shows that neighboring interfacial water molecules, in contact with the bulk liquid, form dimers, in clear contrast to what is observed in UHV experiments.

\section{Results and Discussion}

The rutile surface was first prepared in UHV by sputtering/annealing cycles to produce a $(1 \times 1)$ structure, ${ }^{[2 a]}$ as verified by low energy electron diffraction (LEED) (Figure 1). After extraction from the UHV chamber, the sample was trans-

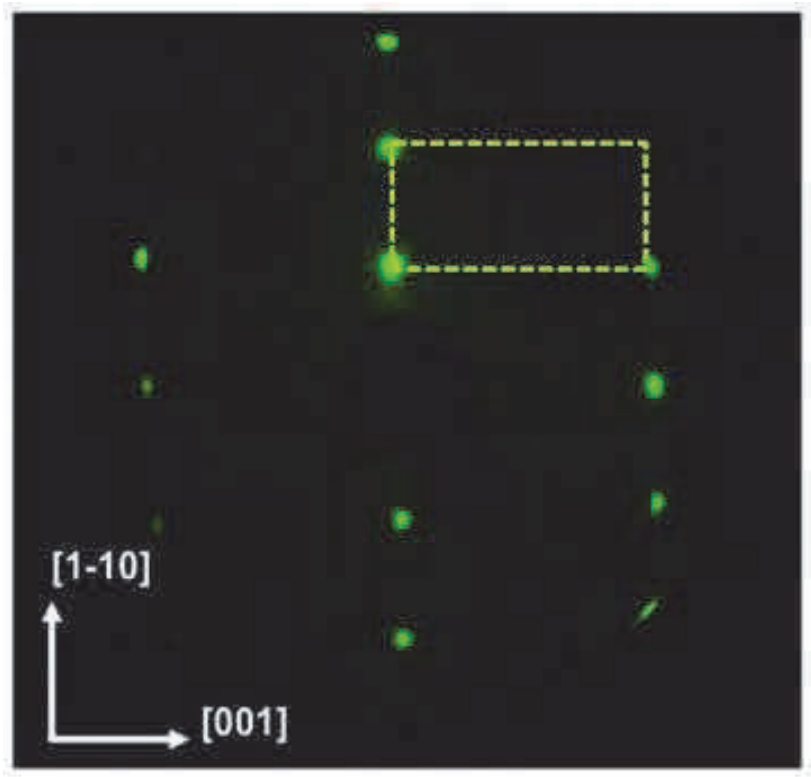

Figure 1. LEED pattern of the $\mathrm{TiO}_{2}(110)$ surface after preparation in UHV (beam energy $E=56 \mathrm{eV}$ ). The rectangular $1 \times 1$ unit cell is highlighted by the dashed yellow lines.

ferred (see the Experimental Section) into the liquid cell and immersed in high-purity water.

A typical large-scale in situ STM ${ }^{[31]}$ image of the immersed sample is shown in Figure 2. The surface consists of distinct, flat terraces, separated by monatomic steps of the expected height $(3.2 \pm 0.5 \AA) .{ }^{[2 a]}$ This morphology closely resembles the one typically observed when similarly-prepared $\mathrm{TiO}_{2}(110)$ surfaces are imaged with STM directly in UHV. ${ }^{[2 a]}$ Even after two days of immersion in water this structure had not changed and did not show evidence of dissolution or erosion.

A representative high-resolution STM image, recorded in bulk water on one of the terraces is shown in Figure 3A. The image quality is remarkably good considering the dynamic fluctuations of the surrounding water at room temperature. Alternating bright and dark rows that run along the [001] direction are visible. An apparent long-range height modulation is also visible, that results in nm-sized brighter areas (lower left part and upper central part of Figure 3A), which were observed in all regions monitored across the surface. Similar features are often reported in UHV-STM studies, and mostly attributed to local variations of the electronic structure (caused by positively charged impurities below the surface, ${ }^{[32]}$ or Ar implanted in the sputtering process ${ }^{[33]}$. From the height profile in Figure 3B, taken along the $[1 \overline{1} 0]$ direction (perpendicular to the rows) the distance between adjacent bright (or dark) rows is $7.0 \pm 0.5 \AA$. This value matches the $\times 1$ periodicity along the $[1 \overline{1} 0]$ direction measured in UHV. ${ }^{[2 a]}$ Also the corrugation perpendicular to the rows $(0.3 \pm 0.1 \AA)$ compares well with values measured with STM in UHV. ${ }^{[2 a]}$ On the contrary, the corrugation we measure along the rows, i.e., the [001] direction, is considerably higher than what is observed along the same direction in UHV by 


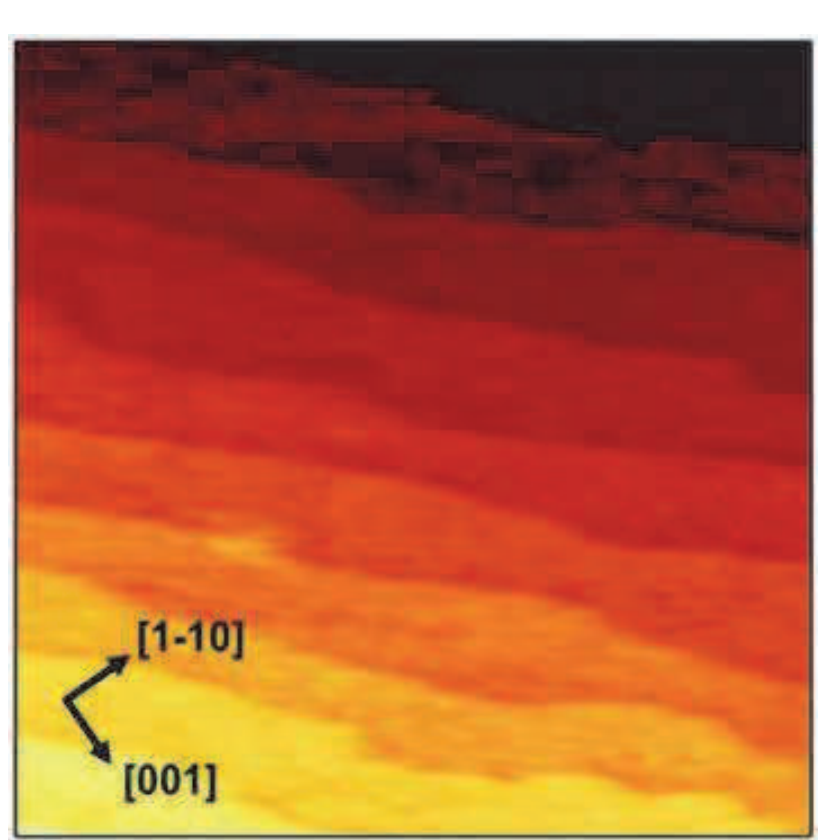

Figure 2. Surface morphology of rutile $\mathrm{TiO}_{2}(110)$ immersed in high-purity liquid water. In situ STM image $\left(450 \times 450 \mathrm{~nm}^{2}\right.$, $V_{\text {bias }}=-0.7 \mathrm{~V}$ (empty states), $\left.l_{\text {tunnel }}=1 \mathrm{nA}\right)$. The measured step height value is $3.2 \pm 0.5 \AA$.

STM (typically less than $0.1 \AA$ for the clean surface in UHV ${ }^{[34]}$ against $0.4 \pm 0.1 \AA$ in our experiments).

Interestingly, weak protrusions, visible along the bright rows and separated by twice (within the experimental accuracy) the known value of the $\times 1$ periodicity along the [001] direction (3.0 A) suggest a $\times 2$ superstructure (see the height profile in Figure $3 \mathrm{C}$, and the inset of Figure $3 \mathrm{~A}$ ). This result is well confirmed by the fast Fourier transform (FFT) in Figure 4, where the $\times 2$ structural motif appears as a true periodicity in the lattice. The white full circles in the FFT indicate maxima from the periodic spacing between the rows (i.e., $6.5 \AA$ along [110] see Figure 5A, where the $1 \times 1$ unit cell is represented). The same periodicity (white dashed circles) is repeated at a separation that corresponds to about $6 \AA$ in real space, i.e., at a separation of twice the unit cell vector along [001] (see for comparison the unit cell reported in Figure 5A). From the alignment of the spots one could, in principle, determine whether this periodicity corresponds to a $\mathrm{p}(2 \times 1)$ (Figure $4 \mathrm{~B}$ ) or a $\mathrm{c}(2 \times 2)$ (Figure 4C) superstructure. However, drift along the slow-scan direction (the vertical-direction in Figure 3A) leads to slight image distortions that prevent a confident assignment.

This detected periodic superstructure is at variance with the $(1 \times 1)$ LEED pattern obtained from the sample after preparation in UHV before immersion in water (Figure 1). Since we rule out surface contamination as possible origin of this new superstructure (see the Experimental Section), we assign the observed periodicity as due to the first, i.e., interfacial ML of the bulk water phase in direct contact with the surface. Water molecules that are bound to exposed cations on rutile $\mathrm{TiO}_{2}(110)$ are typically imaged as bright protrusions in UHV STM. ${ }^{[10,12,35]}$
A

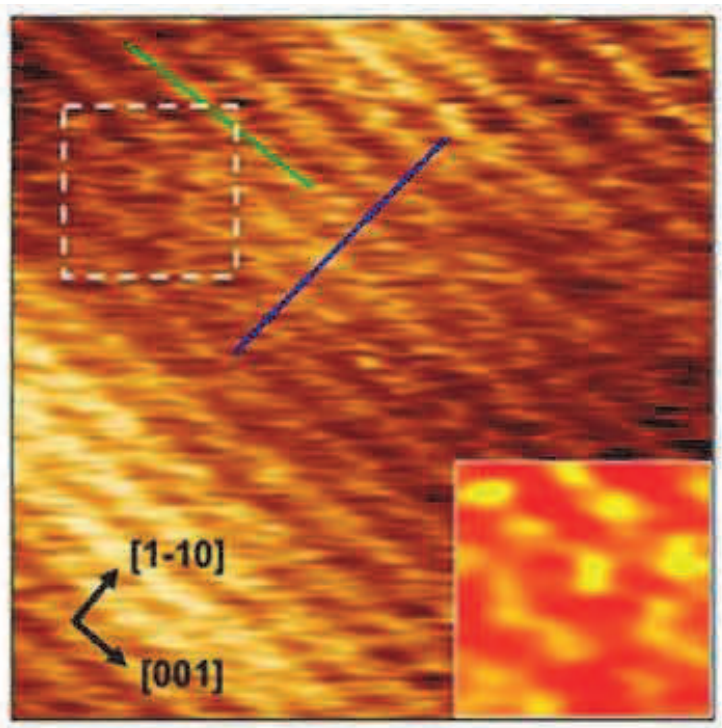

B
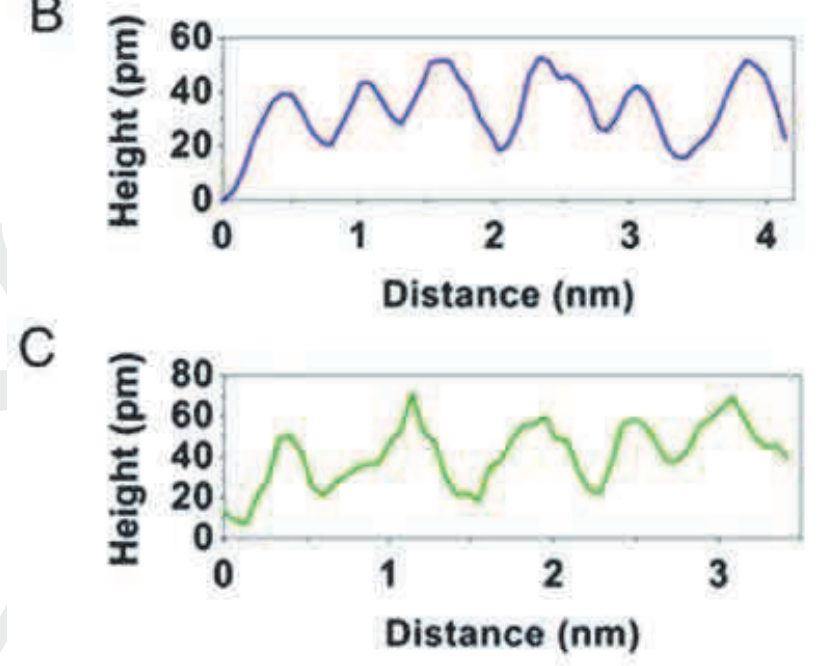

Figure 3. A) STM image $\left(10 \times 10 \mathrm{~nm}^{2}, V_{\text {bias }}=-0.7 \mathrm{~V}\right.$ (empty states), $I_{\text {tunnel }}=1 \mathrm{nA}$ ) of rutile $\mathrm{TiO}_{2}(110)$ in high-purity liquid water. (Inset): Reproduction (with a different color scale) of the $2.5 \times 2.5 \mathrm{~nm}^{2}$ region in the white dashed square. B) Height profile along the blue line in (A) (perpendicular to the rows). C) Height profile along the green line in (A) (parallel to the rows).

Experimental work and theoretical studies agree that water binds strongly to the exposed fivefold coordinated $\mathrm{Ti}_{5 \mathrm{c}}$ atoms of the rutile $\mathrm{TiO}_{2}(110)$ surface in the liquid, tightly enough to be stable under the scanning STM tip. The results in Figures 3 and 4 would then indicate that the structure of the adsorbed layer possesses a $\times 2$ periodicity along the [001] direction.

In parallel with these experiments we have performed DFTMD simulations for the rutile $\mathrm{TiO}_{2}(110)$ /water interface considering both single-monolayer and bulk liquid models. The results of the simulations are presented in Figure 5.

A snapshot of the $\mathrm{TiO}_{2}$ /bulk liquid interface is sketched in Figure 5A. The water molecules directly adsorbed on the $\mathrm{TiO}_{2}$ 


\section{ADVANCED
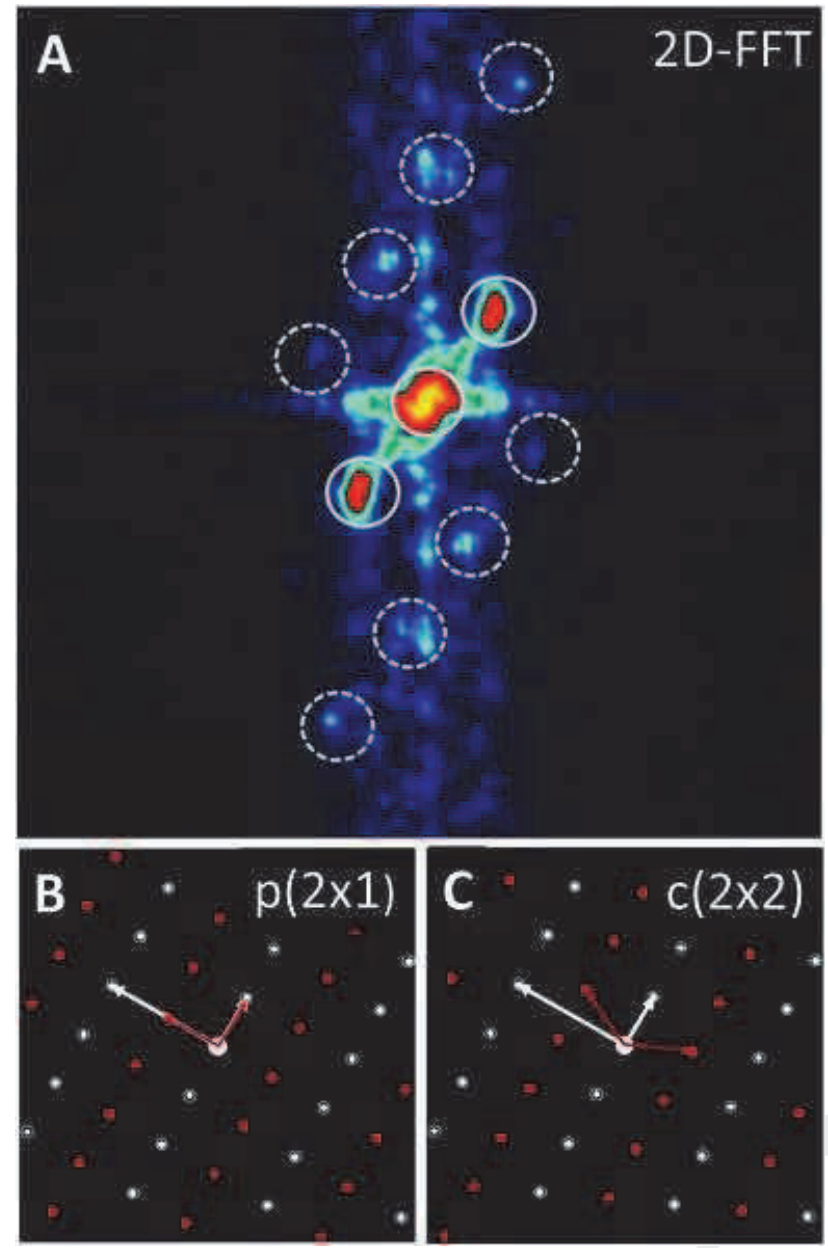

Figure 4. A) 2D fast Fourier transform (2D-FFT) of the STM image reported in Figure 3A. The FFT map shows the long-range periodicities of the surface. Solid line white circles correspond to the $1 \times 1$ periodicity of the $\mathrm{TiO}_{2}(110)$ substrate along the $[1 \overline{1} 0]$ direction between rows visible in the real-space image. Dashed white circles show a superstructure with $\times 2$ periodicity along the rows parallel to the [001] direction. B,C) Calculated reciprocal lattice pattern for the $\mathrm{TiO}_{2}(110) 1 \times 1$ substrate (white spots) with two different surface superstructures: $(B) p(2 \times 1)$ or $(C)$ $c(2 \times 2)$ (red spots). The unit cell vectors of the reciprocal lattice are reported for the $\mathrm{TiO}_{2} 1 \times 1$ substrate (white lines) and for the two superstructures (red lines).

surface are located on the rows of $\mathrm{Ti}_{5 \mathrm{c}}$ atoms running along the crystallographic [001] direction (separated by twofold coordinated $\mathrm{O}$ atoms, so-called "bridging" $\mathrm{O}_{\mathrm{br}}$ rows). Along these Ti rows, alternating short and long $\mathrm{O}-\mathrm{O}$ distances are observed between neighboring water molecules. Whereas a hydrogen bond (HB) is present between the two nearest neighbors that are closer, this is not the case for the two molecules that are further apart. The donating molecule in the water dimer is typically lying flat, while the acceptor molecule of the dimer has its hydrogen atoms pointing up. This dimerization is quantified in Figure 5B, which shows the distribution of O-O distances.
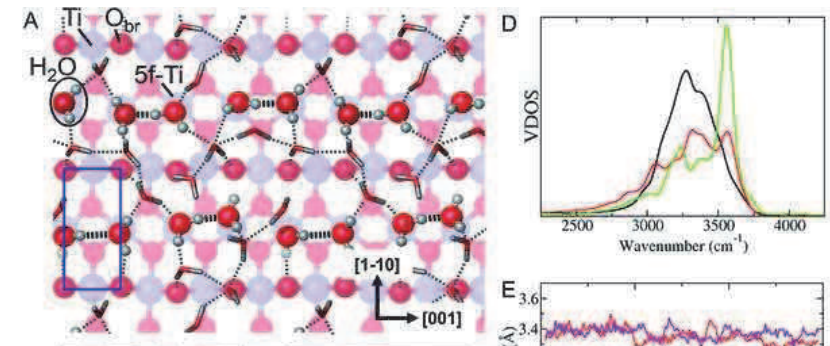

B
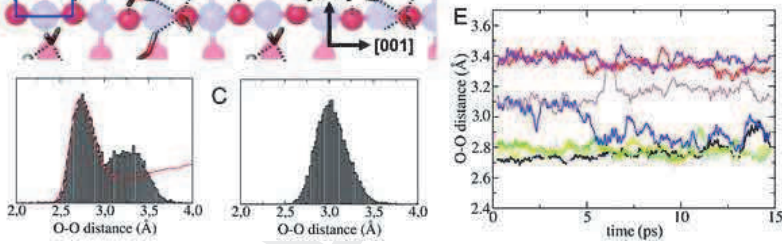

Figure 5. Dimerization of neighboring water molecules in DFTMD simulations. A) Snapshot of the DFT-MD simulation of the rutile $\mathrm{TiO}_{2}$ (110)/bulk-water interface; see the Supporting Information for a movie. Dashed lines represent hydrogen bonding $(\mathrm{HB})$ interactions, shown in thick black lines between water molecules of the first layer (ball-and-stick representation) and in thin gray lines for those involving water molecules from the bulk (sticks only). The blue rectangle indicates the rutile $\mathrm{TiO}_{2}(110)$ $(1 \times 1)$ unit cell $\left(3.0 \times 6.5 \AA^{2}\right)$. A single water molecule is highlighted in the black oval. B) Histogram of nearest neighbor $\mathrm{O}-\mathrm{O}$ distances for oxygen belonging to the first layer of water molecules at the rutile $\mathrm{TiO}_{2}(110)$ /bulk-water interface. The red line shows the $\mathrm{O}-\mathrm{O}$ radial distribution function of bulk water ${ }^{[36]}$ for comparison. C) Corresponding histogram of nearest neighbor O-O distances for a single ML of adsorbed water molecules on rutile $\mathrm{TiO}_{2}(110)$ at $300 \mathrm{~K}$. D) Computed vibrational density of states (VDOS) of the $\mathrm{OH}$ stretch band for the first layer of water molecules at the solid/liquid interface (red), bulk liquid water (black), and a single monolayer of adsorbed molecules (green). E) Time evolution of the $\mathrm{O}-\mathrm{O}$ distance (running average over $0.5 \mathrm{ps}$ ) between molecules of the first layer in bulk water shows long-living dimer species.

For the water molecules on the $\mathrm{TiO}_{2}$ surface immersed in the liquid, this distribution is clearly bimodal, with the peak at short distances (centered at around $2.73 \AA$ ) matching the location and shape of the first peak of the $\mathrm{O}-\mathrm{O}$ radial distribution function in bulk liquid water. ${ }^{[36]}$ The positions of the dimers in the adjacent rows are shifted (Figure 5A). The data are not sufficient to judge if the resulting configuration (which, in case of long-range order, would correspond to a $\mathrm{c}(2 \times 2)$ periodicity $)$ is obtained coincidentally, or if it is energetically favored. Figure $5 \mathrm{C}$ shows that the situation is very different in the single monolayer case (i.e., an adsorbed layer that is not immersed in water, typical situation of UHV studies). Here, the distribution of O-O distances is unimodal, with a maximum at about $3 \AA$, i.e., the $\mathrm{Ti}_{5 c}-\mathrm{Ti}_{5 c}$ separation.

We note that in our simulations one out of 16 surface water molecules dissociates spontaneously in a process that involves a central water molecule accepting two HBs from neighboring surface water molecules (forming a trimer), and donating its proton, via a bulk water molecule, to a bridge-bonding surface oxygen. However, as the energetics of this process is delicate 
and finite size effects tend to favor dissociation, we have presented in Figure 5 the data from the slab surface on which all molecules remained intact. Results for the case in which some molecules are dissociated are qualitatively similar (see Figure $\mathrm{S} 1$ in the Supporting Information).

In earlier UHV work, water-water pairing was observed also for a single water layer on $\mathrm{ZnO}(10 \overline{10}),{ }^{[37]}$ where it leads to the dissociation of every other water molecule. This dissociation was dynamic, however, with the consequence that in UHV STM the observed corrugation (which is due to upward-pointing $\mathrm{H}$ ) was reduced if dissociation happened faster than the time scale of the STM measurement. A similar situation (i.e., fluctuations with frequent associations/dissociations and rearrangements of the dimers) could account for the relatively weak and noisy appearance of the $\times 2$ periodicity in Figure 3 .

While at low coverage water molecules were observed to form dimers on the rutile $\mathrm{TiO}_{2}(110)^{[12]}$ as well as on the isostructural $\mathrm{RuO}_{2}(110)^{[35]}$ (where it also leads to dissociation), this is not the case in a full layer of water on rutile $\mathrm{TiO}_{2}(110)$ in UHV: no dimerization was apparent in STM studies when the surface was fully covered with water from the gas phase at low temperatures. ${ }^{[10,11]}$ This is consistent with our MD results: pairing is not present for a single adsorbed monolayer (Figure 5C, see also ref. $\left.{ }^{[38]}\right)$. The molecule-molecule separation in a dimer at the interface with bulk water is similar to that of HBs typical for liquid water but smaller than in the monolayer-only structure. This difference is also reflected in the computed vibrational spectra of these systems (Figure 5D), where the intensity of the peak at high wavenumbers, corresponding to the weak hydrogen bonds between water molecules of the monolayer-only system, ${ }^{[38]}$ is strongly reduced and broadened in the bulk water system. This provides a molecular picture for the distinct change in the vibrational structure that has been observed in experiments ${ }^{[38]}$ (conducted in UHV at low temperatures) at the transition from low coverage to thicker layers.

Our experimental findings are consistent with these computational results, although the timescale of the STM experiment (about $1 \mathrm{~ms}$ per pixel) is orders of magnitude longer than what can be achieved in first-principles MD simulations. As shown in Figure 5E, some hydrogen-bonded dimers persisted during the full simulation run (tens of picoseconds). The agreement between theory and experiment suggests that dimers could be stable for much longer.

\section{Conclusions}

In conclusion, we report for the first time high-resolution in situ STM images of the $\mathrm{TiO}_{2}(110)$ surface in direct contact with bulk liquid water. In our experimental conditions, the morphology of the oxide surface proved to be stable in water and no dissolution or erosion effects have been observed. The rutile (110) surface shows characteristic steps of monoatomic height, with step edges running along the [001] direction, as in the UHV experiments. High-resolution STM images show bright and dark rows oriented along the [001] direction and spaced with the $\times 1$ periodicity corresponding to the separation of the $\mathrm{Ti}$ and $\mathrm{O}$ rows on the $\mathrm{TiO}_{2}$ rutile (110) surface. A new $\times 2$ periodicity, however, has been observed along these rows, after the interaction with bulk water. This is assigned to an ordering of interfacial water molecules along the rows in the presence of the bulk liquid phase. DFT-MD simulations provide strong evidence that a single adsorbed layer of water on rutile $\mathrm{TiO}_{2}(110)$ changes its structure in the presence of the bulk liquid. While water in the first ML interacts mostly with the undercoordinated $\mathrm{Ti}_{5 \mathrm{c}}$ cations, the presence of the additional layers of bulk water causes neighboring molecules to form hydrogen-bonded pairs.

The striking difference of the molecular ordering at the interface between liquid water and rutile $\mathrm{TiO}_{2}(110)$ surface with respect to the UHV case is clearly evidenced in our results. It convincingly demonstrates that in situ experiments under realistic conditions accompanied by theoretical simulations are the necessary methodological route for understanding the role of $\mathrm{TiO}_{2} /$ water interfaces in real applications, for example the photocatalysis of $\mathrm{TiO}_{2}$ surfaces and nanoparticles in aqueous solution.

\section{Experimental Section}

The rutile $\mathrm{TiO}_{2}$ (110) single crystal (Pi-Kem LTD) has been investigated with a STM in a liquid cell. ${ }^{[3]}$ The surface was prepared in UHV (pressure $<1 \times 10^{-10}$ mbar) by standard preparation methods:[2a] cycles of $\mathrm{Ar}^{+}$ion sputtering $(1 \mathrm{keV})$ and annealing to $\mathrm{T}$ in the range $900-950 \mathrm{~K}$. In LEED the as prepared surface showed the expected $(1 \times 1)$ periodicity. ${ }^{[2 a]}$ After preparation, the sample was taken out of the vacuum and transferred under Ar flux in the STM liquid cell,, ${ }^{[31]}$ filled with high-purity water (Milli-Q purification system, resistivity 18.2 $\mathrm{M} \Omega \mathrm{cm}$ ). The water was degassed by a flux of high purity $\mathrm{Ar}$ for $1 \mathrm{~h}$ before filling the cell. For the STM measurements the sample was grounded and a bias voltage was applied to the tip: thus empty states are imaged at a negative bias potential. The STM tips (tungsten) were prepared by chemical etching (2 M $\mathrm{KOH}$ solution) and then coated with a drop of hot glue. STM images were analyzed with the WS $\times$ M software. ${ }^{[39]}$

Great care (in terms of water purity, sample handling and cell cleanliness standard) has been taken to ensure that observed atomic-scale structures are not due to contamination. To test the effectiveness of the applied cleanliness procedure, various metallic surfaces were immersed in the same cell and then checked in situ with the EC-STM. All these samples displayed the characteristic structure of the corresponding clean surface. ${ }^{[31,40,41]}$

We have performed DFT-MD simulations for the $\mathrm{TiO}_{2}(110) /$ water interface. The calculations were performed with the CP2K program using the Gaussian and plane-waves approach. ${ }^{[2,43]}$ The model employed is based on a five O-Ti-O trilayer slab, using a $4 \times 2$ supercell, separated by $15 \AA$, filled with 71 water molecules, as employed in earlier work. ${ }^{[21,44]}$ Here the Perdew-Burke-Ernzerhof (PBE) functional ${ }^{[45]}$ with the Grimme D3 dispersion correction was employed. ${ }^{[46]}$ For bulk liquid water, it is important to use a dispersion correction to obtain the correct density or pressure. ${ }^{[44]}$ The simulation temperature was $300 \mathrm{~K}$, the length $40 \mathrm{ps}$, and the last $15 \mathrm{ps}$ 


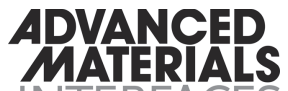 1}

were analyzed and reported. Similar structures formed in a second simulation using only the PBE functional at $330 \mathrm{~K}$, and a 5 ps PBE0-D3 simulation at $300 \mathrm{~K}$, suggesting the observed structures are insensitive to the precise computational setup.

\section{Supporting Information}

Supporting Information is available from the Wiley Online Library or from the author.

\section{Acknowledgements}

The authors gratefully acknowledge "Polo Solare Organico Regione Lazio" for partial support. U.D. acknowledges support from the ERC Advanced Grant "OxideSurfaces." J.V. acknowledges financial support by the European Union FP7 in the form of an ERC Starting Grant under Contract No. 277910. Calculations were enabled by the Swiss National Supercomputer Centre (CSCS) under Project ID ch5. The authors acknowledge valuable assistance and technical support by M. Iannilli, D. Pecchi, and G. Vitali (Dipartimento di Fisica, Rome "Tor Vergata").

Received: May 11, 2015 Revised: July 30, 2015 Published Online: MM DD, YYYY

[1] a) A. Fujishima, K. Honda, Nature 1972, 238, 37; b) M. A. Henderson, Surf. Sci. Rep. 2011, 66, 185; c) S. J. Bao, C. M. Li, J. F. Zang, X. Q. Cui, Y. Qiao, J. Guo, Adv. Funct. Mater. 2008, 18, 591; d) M. Grätzel, Inorg. Chem. 2005, 44, 6841.

[2] a) U. Diebold, Surf. Sci. Rep. 2003, 48, 53; b) C. L. Pang, R. Linsday, G. Thornton, Chem. Rev. 2013, 113, 3887.

[3] G. Ketteler, S. Yamamoto, H. Bluhm, K. Andersson, D. E. Starr, D. F. Ogletree, H. Ogasawara, A. Nilsson, M. Salmeron, J. Phys. Chem. C 2007, 111, 8278.

[4] a) P. Fenter, N. C. Sturchio, Prog. Surf. Sci. 2004, 77, 171; b) M. Komiyama, M. Gu, Appl. Surf. Sci. 1997, 120, 125; c) H. Uetsuka, A. Sasahara, H. Onishi, Langmuir 2004, 20, 4782; d) A. Sasahara, M. Tomitori, J. Vac. Sci. Technol. B 2010, 28, C4C5.

[5] a) C. L. Pang, R. Linsday, G. Thornton, Chem. Soc. Rev. 2008, 37, 2328; b) A. G. Thomas, K. L. Syres, Chem. Soc. Rev. 2012, 41, 4207.

[6] U. Aschauer, A. Selloni, Phys. Rev. Lett. 2011, 106, 166102.

[7] S. Wendt, J. Matthiesen, R. Schaub, E. K. Vestergaard, E. Lægsgaard, F. Besenbacher, B. Hammer, Phys. Rev. Lett. 2006, 96, 066107.

[8] H. H. Kristoffersen, J. Ø. Hansen, U. Martinez, Y. Wei, J. Matthiesen, R. Streber, R. Bechstein, E. Lægsgaard, F. Besenbacher, B. Hammer, Phys. Rev. Lett. 2013, 110, 146101.

[9] R. Schaub, P. Thostrup, N. Lopez, E. Lægsgaard, I. Stensgaard, J. K. Nørskov, F. Besenbacher, Phys. Rev. Lett. 2001, 87, 266104.

[10] I. Brookes, C. Muryn, G. Thornton, Phys. Rev. Lett. 2001, 87, 266103.

[11] J. Lee, D. C. Sorescu, X. Y. Deng, K. D. Jordan, J. Phys. Chem. Lett. 2013, 4, 53.
[12] J. Matthiesen, J. Ø. Hansen, S. Wendt, E. Lira, R. Schaub, E. Lægsgaard, F. Besenbacher, B. Hammer, Phys. Rev. Lett. 2009, 102, 226101.

[13] M. A. Henderson, Langmuir 1996, 12, 5093.

[14] S. Wendt, R. Schaub, J. Matthiesen, E. Vestergaard, E. Wahlström, M. Rasmussen, P. Thostrup, L. Molina, E. Lægsgaard, I. Stensgaard, Surf. Sci. 2005, 598, 226.

[15] C. Pang, A. Sasahara, H. Onishi, Q. Chen, G. Thornton, Phys. Rev. B 2006, 74, 073411.

[16] O. Bikondoa, C. L. Pang, R. Ithnin, C. A. Muryn, H. Onishi, G. Thornton, Nat. Mater. 2006, 5, 189.

[17] C. Sun, L.-M. Liu, A. Selloni, G. Q. M. Lu, S. C. Smith, J. Mater. Chem. 2010, 20, 10319.

[18] G. Ketteler, S. Yamamoto, H. Bluhm, K. Andersson, D. E. Starr, D. F. Ogletree, H. Ogasawara, A. Nilsson, M. Salmeron, J. Phys. Chem. C 2007, 111, 8278.

[19] L. Walle, D. Ragazzon, A. Borg, P. Uvdal, A. Sandell, Surf. Sci. 2014, 621, 77 .

[20] L.-M. Liu, C. Zhang, G. Thornton, A. Michaelides, Phys. Rev. B 2010, 82, 161415 .

[21] J. Cheng, J. VandeVondele, M. Sprik, J. Phys. Chem. C 2014, 118,5437

[22] M. Raju, S.-Y. Kim, A. C. T van Duin, K. A. Fichthorn, J. Phys. Chem. C 2013, 117, 10558.

[23] N. Kumar, P. R. C. Kent, D. J. Wesolowski, J. D. Kubicki, J. Phys. Chem. C 2013, 117, 23638.

[24] R. S. Kavathekar, N. J. English, J. M. D. MacElroy, Chem. Phys. Lett. 2012, 554, 102.

[25] J. Cheng, M. Sprik, J. Chem. Theory Comput. 2010, 6, 880.

[26] P. Fenter, N. C. Sturchio, Prog. Surf. Sci. 2004, 77, 171.

[27] Z. Zhang, P. Fenter, N. C. Sturchio, M. J. Bedzyk, M. L. Machesky, D. J. Wesolowski, Surf. Sci. 2007, 601, 1129.

[28] D. J. Wesolowski, J. O. Sofo, A. V. Bandura, Z. Zhang, E. Mamontov, M. Předota, N. Kumar, J. D. Kubicki, P. R. Kent, L. Vlcek, Phys. Rev. B 2012, 85, 167401.

[29] H. Uetsuka, A. Sasahara, H. Onishi, Langmuir 2004, 20, 4782.

[30] A. Sasahara, M. Tomitori, J. Vac. Sci. Technol. B 2010, 28, C4C5.

[31] M. Wilms, M. Kruft, G. Bermes, K. Wandelt, Rev. Sci. Instrum. 1999, 70, 3641.

[32] M. Batzill, K. Katsiev, D. J. Gaspar, U. Diebold, Phys. Rev. B 2002, 66, 235401.

[33] D. V. Potapenko, Z. Li, J. W. Kysar, R. M. Osgood, Nano Lett. 2014, 14, 6185.

[34] D. Novak, E. Garfunkel, T. Gustafsson, Phys. Rev. B 1994, 50, 5000.

[35] R. Mu, D. C. Cantu, X. Lin, V.-A. Glezakou, Z. Wang, I. Lyubinetsky, R. Rousseau, Z. Dohna'lek, J. Phys. Chem. Lett. 2014, 5, 3445 .

[36] M. Del Ben, M. Schönherr, J. Hutter, J. VandeVondele, J. Phys. Chem. Lett. 2013, 4, 3753.

[37] O. Dulub, B. Meyer, U. Diebold, Phys. Rev. Lett. 2005, 95, 1.

[38] G. A. Kimmel, M. Baer, N. G. Petrik, J. VandeVondele, R. Rousseau, C. J. Mundy, J. Phys. Chem. Lett. 2012, 3, 778.

[39] I. Horcas, R. Fernandez, J. Gomez-Rodriguez, J. Colchero, J. Gómez-Herrero, A. Baro, Rev. Sci. Instrum. 2007, 78, 013705.

$[40]$ a) P. Broekmann, M. Wilms, A. Spaenig, K. Wandelt, Prog. Surf. Sci. 2001, 67, 59; b) B. Obliers, M. Anastasescu, P. Broekmann, K. Wandelt, Surf. Sci. 2004, 573, 47; c) S. Hümann, N.T.M. Hai, P. Broekmann, K. Wandelt, H. Zajonz, H. Dosch, F. Renner, J. Phys. Chem. B 2006, 110, 24955; d) D.-T. Pham, S.-L. Tsay, K. Gentz, C. Zoerlein, S. Kossmann, J.-S. Tsay, B. Kirchner, K. Wandelt, P. Broekmann, J. Phys. Chem. C 2007, 111, 16428 
www.AdvMatInterfaces@wiley-vch.de

[41] C. Goletti, G. Bussetti, A. Violante, B. Bonanni, M. Di Giovannantonio, G. Serrano, S. Breuer, K. Gentz, K. Wandelt, J. Phys. Chem. C 2014, 119, 1782.

[42] J. VandeVondele, M. Krack, F. Mohamed, M. Parrinello, T. Chassaing, J. Hutter, Comput. Phys. Commun. 2005, 167, 103.

[43] The CP2K developers group, http://www.cp2k.org/ (accessed: 2014).

[44] J. Cheng, X. Liu, J. A. Kattirtzi, J. VandeVondele, M. Sprik, Angew. Chem. Int. Ed. 2014, 53, 12046.

[45] J. Perdew, K. Burke, M. Ernzerhof, Phys. Rev. Lett. 1996, 77, 3865.

[46] S. Grimme, J. Antony, S. Ehrlich, H. Krieg, J. Chem. Phys. 2010, $132,154104$. 